\title{
Surgical oophorectomy: a primary option in adjuvant treatment of premenopausal breast cancer
}

\section{Author:}

Richard R. Love, M.D., M.S.*

*From the Amader Gram Cancer Care and Research Center, Rampal, Bangladesh; and the Department of Mathematics, Statistics and Computer Science, Marquette University, Milwaukee, WI, U.S.A.

\section{Correspondence address:}

2708 Columbia Road, Madison, WI 53705 U.S.A. Tel: 608 238-7881

E-mail: richardibcrf@gmail.com

No funding

Short title: Surgical oophorectomy for breast cancer

Precis: An important issue for global women's health is that surgical oophorectomy should be considered a primary breast cancer adjuvant treatment option.

\begin{abstract}
Globally, there are 500,000 annual new cases of hormone-sensitive breast cancer in premenopausal women; 400,000 of these occur in women in low and middle income countries (LMIC). With optimal adjuvant therapy, fully one third more than the half of women who might survive with primary surgery alonemore than $80 \%$, could survive 10 years. Affordability and practicality of treatment profoundly affect global practice and these do not characterize the current standards of care - LHRH agonist plus tamoxifen or an aromatase inhibitor.
\end{abstract}

Surgical oophorectomy plus tamoxifen is a safe, efficient and affordable, patient-centered and equitable adjuvant treatment, long considered equivalent to that from LHRH agonist plus tamoxifen treatment. New data additionally suggest that women, who are in extended follicular phase of their cycles at the time of surgery, benefit much less from this treatment, and women who are in normal progesterone-confirmed follicular or luteal phases, benefit more than unselected or LHRH-treated women. New data also show that surgical oophorectomy plus tamoxifen is associated with no loss of bone mineral density at the hip, and loss for only a year in the lumbar spine.

The dominance of a business model for medicine, the narrow perspective and single metric-efficacy focus of guideline creation, and limited understanding of the practice of medicine for poor women worldwide, appear to be contributing to the social injustice of not offering women the option of surgical oophorectomy instead of LHRH treatment. 100,000 women a year could be saved. Further research investigating outcomes associated with the timing of surgical oophorectomy is strongly justified. 
Addressing women's health issues successfully often involves understanding and explaining to patients the powerful hormonal biology - not unnoticed by women of course - that characterizes particularly the premenopausal years. This has been obviously true with respect to breast cancer, where critical parameters of ovarian function are related to disease development (age at menarche and age at menopause, specifically), and the presence and relative concentrations of estrogen and progesterone protein receptors in twothirds of invasive tumors are strong predictors of therapeutic responsiveness to hormonal change, and indicators of disease chronicity. Globally, there are 500,000 annual new cases of hormonesensitive breast cancer in premenopausal women; 400,000 of these cases are among women in low- and middle-income countries (LMIC). With optimal hormonal adjuvant therapy, fully one third more than the half of women who might survive with primary surgery alone-more than $80 \%$, could survive 10 years. (1). Over the last 25 years, while the systemic therapy management of such hormone-sensitive breast cancer has become medical oncology work in high-income countries, newer nuanced biological data about the role of surgical oophorectomy, and the major roles of obstetrics and gynecology specialists world-wide in breast cancer care, combined with increasing concern about equity in health care, suggest that ob-gyn specialists should now play an even more central role in breast cancer care.

With the publication in the early 90s of a meta-analysis showing that adjuvant ovarian ablation (mostly by surgery) was associated with better outcomes in women with breast cancer, the role of this intervention (which for all of the early part of the century had been an acknowledged treatment for metastatic breast cancer) came back into the fore after a 20-year period of obscurity (2). In that interval, LHRH agonists had been developed and short-term adjuvant treatment with these ovarian-function-suppressing agents, in patients with hormone receptor positive tumors, was adopted as a less invasive, and reversible approach which has been considered equivalent in efficacy. During the 90s however, it became increasingly clear that hormone receptor-positive breast cancer is a chronic disease and longer term ovarian suppression treatment of at least 5 years was demonstrated to be more effective, and is now the standard (3). Combined hormonal therapies with an LHRH agonist and an endocrine therapy such as tamoxifen (a specific breast cancer cell estrogen receptor blocker) or an aromatase inhibitor (which lowers systemic tissue-produced estrogen levels) have been found more effective than single hormonal therapies, and are now favored, particularly in higher-risk patients (3). The situation regarding treatment after 5 years is unsettled; continuing tamoxifen alone is one recommendation.

Contemporary with the LHRH/ endocrine therapy (with tamoxifen or an aromatase inhibitor) studies of the last 20 years, studies of surgical oophorectomy and tamoxifen have been pursued. An initial trial in Vietnam confirmed the safety and efficacy of adjuvant surgical oophorectomy and tamoxifen $(\mathrm{SO}+\mathrm{T})$ for 5 years, demonstrating an absolute improvement of $33 \%$ in overall survival at 10 years, and a risk reduction of $65 \%$ compared with no systemic adjuvant treatment (1). The suggestion in secondary analyses that the time of surgical oophorectomy in the menstrual cycle impacted survivals in this trial led to two 
subsequent adjuvant and metastatic studies investigating this possibility $(4,5)$. In the adjuvant trial, randomized women in historical luteal phase, but with low progesterone levels consistent with prolonged follicular status, benefited little from $\mathrm{SO}+\mathrm{T}$, while women in historical follicular and luteal phases with respectively confirming low and high progesterone levels, benefited more than expected--their disease free and overall survivals were better than for the average for all women treated with $\mathrm{SO}+\mathrm{T}$, and thus by extension better than the average for women treated with LHRH $+\mathrm{T}$. In the metastatic trial, the same subgroup-of luteal phase history-low progesterone women-- had poorer median survival by 10 months less than either the low progesterone-confirmed follicular history, or high progesterone-confirmed luteal history women. These consistent results from two studies suggest that if among an average, but high-risk group of women with operable breast cancer receiving SO $+\mathrm{T}$, (without paying any attention to their menstrual cycle history and blood levels of progesterone), $65 \%$ have no recurrence in 5 years, if patients have their SO in the first half of their menstrual cycles by history and with confirmation showing low progesterone blood levels, $72 \%$ will have no recurrence in 5 years (6). These results are consistent with those from a trial in which parental progesterone perioperatively was associated with better outcomes in axillary node positive patients, and with a mathematical model of the major consequences of both positive and negative "minor" peri-operative changes $(7,8)$. Immediate benefit from oophorectomy in the selected cases is not surprising given the analogous immediate benefit from orchiectomy seen in men with metastatic prostate cancer.
Beyond the possible greater efficacy of SO over LHRH agonist treatment in selected women, two additional characteristics of SO make a compelling case for its reintroduction into the global treatment armamentarium. A large study of the impact of $\mathrm{SO}+\mathrm{T}$ on bone mineral density showed no evidence of bone loss at the hip over two years and loss in the lumbar spine in the first year only (9). These finding are in contrast to those for all other adjuvant therapies which show continuous loss of bone at both sites (9). This problem has been addressed in high-income countries by administration of bisphosphonate drugs which have their own financial costs and toxicities, particularly of jaw osteonecrosis associated with poor oral hygiene, which is an endemic problem worldwide. The second relevant characteristic of SO is that it can be safety accomplished under the same anesthesia as the primary breast treatment, at minimal direct cost to women throughout the world because surgeries have public health financial support. In contrast, LHRH agonist drugs require major out of pocket expense (in the best of circumstances hundreds of dollars/month) for the majority of women in the world needing such treatment, in addition to which patients incur indirect costs for monthly visits, and blood test confirmation of LHRH biological efficacy. Indeed, the evidence is that the unaffordability and impracticality of LHRH treatment (monthly for 5 years) leads to limited use of this approach in most low- and middleincome countries, and increased mortality, because practical and affordable options have not been promoted. Specifically, a realistic estimate is that if $\mathrm{SO}+\mathrm{T}$ were widely promoted and practiced, 100,000 women per year, who now die of hormone sensitive breast cancer, might be saved. 
Without justification, the recently updated clinical practice guideline of The American Society of Clinical Oncology which influences global practice, now omits any mention of surgical oophorectomy as a treatment option for premenopausal women with hormone receptor positive breast cancer (3). Given now that surgical oophorectomy, compared with LHRH agonist treatment, offers: 1. At least equivalent and possibly greater efficacy in selected patients; 2 . Better bone safety when combined with $\mathrm{T}$; and 3. Better cost-efficacy, value, greater practicality and affordability for women worldwide, women's health specialists should champion surgical oophorectomy as a treatment option. When women have been given this option, they have preferred it to LHRH treatment (10). The dominance of a business model for medicine, the narrow perspective and single metric- efficacy focus of guideline creation, and limited understanding of the practice of medicine for poor women worldwide, appear to be contributing to the social injustice of not offering women the option of surgical oophorectomy instead of LHRH treatment. Women's health specialists can contribute to correcting what Paul Farmer has highlighted-"structural violence" in medicine - the withholding of a very beneficial treatment option for poor women, with consequences of protecting the pharmaceutical industry (11). Finally, the compelling evidence regarding the minimal benefit of surgical oophorectomy in women with prolonged follicular phases, and the correlative greater benefit in normal cycling women, offers a rationale for investigating this widely available surgical treatment further (12). 


\section{References}

1. Love, R.R., Van Dinh, N., Quy, T.T., et al.: Survival after adjuvant oophorectomy and tamoxifen in operable breast cancer in premenopausal women. J Clin Oncol. 2008.26:253-7.

2. Early Breast Cancer Trialists' Collaborative group: Systemic treatment of early breast cancer by hormonal, cytotoxic or immune therapy. Lancet 1992. 339:72-85.

3. Burstein, H.J., Lacchetti, C., Anderson, $\mathrm{H}$. et al: Adjuvant endocrine therapy for women with hormone receptorpositive breast cancer: American Society of Clinical Oncology Clinical Practice Guideline update on Ovarian suppression. J Clin Oncol 2016. 34:1689-1701.

4. Love, R.R., Laudico, A.V., Dinh, N.V., et al.: Timing of adjuvant surgical oophorectomy in the menstrual cycle and disease-free and overall survival in premenopausal women with operable breast cancer. J Nat Cancer Inst 2015 107 (3): djv064 doi: 10.1093/jnci/djv064.

5. Love, R.R., Hossain, S.M., Hussain, M., et al.: Luteal versus follicular phase surgical oophorectomy plus tamoxifen in premenopausal women with metastatic hormone receptor positive breast cancer. Europ J Cancer 2016. 60:107-116. http://dx.doi.org/10.1016/j.ejca.2016.0 3.011 .

6. Love, R.R.: Adjuvant surgical oophorectomy plus tamoxifen in premenopausal women with operable breast cancer: a global treatment option. Clin Breast Cancer 2016.16: 233-7.

http://dx.doi.org/10.1016/j.clbc.2016.0 3.003 .

7. Badwe R, Hawaldar R, Parmar V, et al: Single-injection depot progesterone before surgery and survival in women with operable breast cancer: a randomized controlled trial. J Clin Oncol 2011; 29:2845-51.

8. Baum M, Chaplain MA, Anderson AR, Douek M, Vaidya JS. Does breast cancer exist in a state of chaos? Eur $\mathbf{J}$ Cancer. 1999;35(6):886-91.

9. Love, R.R., Young, G.S., Laudico, A.V., et al: Bone mineral density changes following surgical oophorectomy and tamoxifen adjuvant therapy for breast cancer. Cancer 2013; 119:3746-52.

10. Tevaarwerk, A.J., Wang, M., Zhaon, F. et al.: Phase III Comparison tamoxifen versus tamoxifen plus ovarian function supresssion in premenopausal women with node negative, hormone receptor positive breast cancer (E-3193, INT0142): A trial of the Eastern Cooperative Oncology Group. J Clin Oncol 2014. 32:3948-3958.

11. Farmer, P.: Pathologies of power Health human rights and the new war on the poor. University of California Press, Berkley and Los Angeles, 2005.

12. Love, R.R. Love, S.M.: Peri-operative biology in primary breast cancer: A credible therapeutic target. Breast Cancer Res Treat 2016. 156:411-413. 